\title{
Long non-coding RNA HOTAIR enhances radioresistance in MDA-MB231 breast cancer cells
}

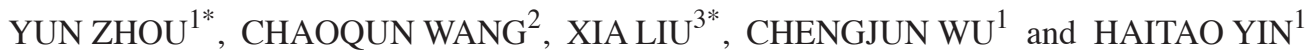 \\ ${ }^{1}$ Department of Radiation Oncology, Xuzhou Central Hospital, The Affiliated Xuzhou Hospital of Medical College \\ of Southeast University, Xuzhou Clinical School of Xuzhou Medical College, Xuzhou, Jiangsu 221009; \\ ${ }^{2}$ College of Basic and Forensic Medicine, Sichuan University, Chengdu, Sichuan 610064; \\ ${ }^{3}$ Department of Pathology, Xuzhou Central Hospital, Xuzhou, Jiangsu 221009, P.R. China
}

Received January 19, 2016; Accepted October 27, 2016

DOI: $10.3892 / \mathrm{ol} .2017 .5587$

\begin{abstract}
The aim of the present study was to investigate the radiosensitizing effects of homeobox (HOX) transcript antisense RNA (HOTAIR) long non-coding RNA on breast cancer tumor cells and examine the underlying mechanisms. Recombinant plasmid vectors containing HOTAIR gene were constructed and MDA-MB231 cells were transfected with these plasmids using liposomes. The cells were treated with radiation and cell apoptosis, proliferation, and double-stranded DNA breaks were examined. HOXD10, phosphorylated AKT (p-AKT) and p-BAD expression levels were measured using western blot analysis. The results showed a higher expression of HOTAIR in advanced tumor cells. HOTAIR efficiently enhanced radioresistance in MDA-MB231 breast cancer cells and accelerated proliferation through the Akt pathway by targeting HOXD10. In conclusion, the findings demonstrated that HOTAIR gene is a valid therapeutic target for the reversal of radiotherapy resistance in breast cancer.
\end{abstract}

\section{Introduction}

Breast cancer is the most frequently occurring cancer among women in 140 countries worldwide. This disease claimed over half a million lives in 2012, and increased $>20 \%$ in incidence and 14\% in mortality since 2008 (1). Postoperative adjuvant therapies including radiotherapy are the main mode of treatment to improve the overall prognosis. Adjuvant therapies

Correspondence to: Dr Haitao Yin, Department of Radiation Oncology, Xuzhou Central Hospital, The Affiliated Xuzhou Hospital of Medical College of Southeast University, Xuzhou Clinical School of Xuzhou Medical College, 199 Jiefang Road, Xuzhou, Jiangsu 221009, P.R. China

E-mail:ndkwof91@163.com

*Contributed equally

Key words: long non-coding RNA, homeobox transcript antisense RNA, breast cancer, radiosensitivity prevent and control the local recurrence of residual malignant tissue, however, the underlying molecular mechanisms remain unknown. Improving knowledge regarding molecular mechanisms involved in this process may be useful for developing effective methods of therapy. In the past few years, thousands of long non-coding RNAs (lncRNAs) were identified and functional roles of lncRNAs in epigenetics have been discussed (2).

The origin of lncRNAs varies from intergenic to coding regions with sense or antisense orientations. Some of these lncRNAs have been linked to oncogenic and tumor suppressor gene regulation. LncRNAs, which play critical roles in cell regulation, are deregulated in a number of cancers, demonstrating oncogenic properties (2). These properties of lncRNAs provide new tumor-related therapeutic targets for successful intervention. HOTAIR is one of the oncogenic lncRNAs.

A group of the genes with HOTAIR-induced polycomb repressive complex 2 (PRC2) occupancy are involved in inhibiting breast cancer progression. HOTAIR-induced PRC2 occupancy involves transcription factors HOXD and PRG1 (coding for progesterone receptors), cell adhesion molecules such as the protocadherin gene family as well as EPHA1 (coding for an ephrin receptor) (3). Overexpression of HOTAIR has been linked to breast cancer metastasis and poor survival rates. Previous studies reported that overexpression of HOTAIR in breast adenocarcinoma cells reduced the apoptotic rate, and increased cell growth, migration, and invasion (4). It has been reported that two types of ncRNAs, miRNA-10b (miR-10b) and homeobox (HOX) RNA, can suppress the translation of the HOXD10 gene. An mRNA encoding a transcriptional repressor was reported to have inhibitory properties affecting genes responsible for cell migration and invasion (5).

The aberrant proliferation of nucleus pulposus cells through derepressing the RhoC-Akt pathway by targeting HOXD10 has also been investigated (6). However, whether a similar functional mechanism and molecular pathways of HOTAIR are involved in the radiotherapy process of breast adenocarcinoma cells remains to be determined. In the present study, we investigated the expression of HOTAIR in breast adenocarcinoma cells and identified that HOTAIR was markedly overexpressed in a cohort of human breast cancer samples and in advanced tumors. The results obtained from the functional analyses of HOTAIR in radiotherapy revealed 
that the upregulation of HOTAIR enhanced the resistance of human breast cancer cells to radiotherapy by downregulating HOXD10 expression. Thus, HOTAIR can be considered as a valid therapeutic target for the reversal of radiotherapy resistance in breast cancer patients.

\section{Materials and methods}

Cell lines. The T47D, MCF-7, SKBR3, BT549, MDA-MB231 and MCF-10A breast cancer cell lines were used in the present study. The T47D and MCF-7 cell lines were grown in RPMI-1640 medium, while the remaining cell lines were grown in DMEM supplemented with $10 \%$ fetal bovine serum (FBS). The cell cultures were incubated at $37^{\circ} \mathrm{C}$ in a humidified environment containing $5 \% \mathrm{CO}_{2}$.

Reverse transcriptase-quantitative PCR. Total RNA from fresh tissues was extracted by TRIzol reagent according to the manufacturer's instructions with minor modifications. The quality and quantity of RNA was determined using a NanoDrop ${ }^{\circledR}$ ND-1000 spectrophotometer. Then, $1 \mu \mathrm{g}$ total RNA from each sample was reverse-transcribed using the RT reagent kit according to the manufacturer's instructions. Real-time PCR was performed to determine the relative expression levels of target genes using the SYBR-Green RT-qPCR kit on the Step One Real-Time PCR Detection system. The level of HOTAIR expression in each sample was normalized to the respective $\beta$-actin expression level. The RNA sequences targeting human HOTAIR were designed according to a previous study (7) and produced by Sangon Biotech Co., Ltd. (Shanghai, China). The sequences used to construct the primers were: HOTAIR sense, 5'-ATA GGC AAA TGT CAG AGG GTT-3' and antisense, 5'-ATT CTT AAA TTG GGC TGG GTC-3'; and $\beta$-actin sense, 5'-AAA GAC CTG TAC GCC AAC AC-3' and antisense, 5'-GTC ATA CTC CTG CTT GCT GAT-3'. The amplification profile was pre-denatured at $95^{\circ} \mathrm{C}$ for $5 \mathrm{~min}$, followed by 40 cycles of denaturation at $95^{\circ} \mathrm{C}$ for $15 \mathrm{sec}$, annealing and extension at $60^{\circ} \mathrm{C}$ for $30 \mathrm{sec}$, respectively. The accuracy of the PCR amplifications was verified by melting curve analyses. The comparative threshold cycle $(\mathrm{Cq})$ method $(\Delta \Delta \mathrm{Cq})$ was employed for the quantification of HOTAIR gene expression. The relative expression amount of HOTAIR to $\beta$-actin was calculated using the equation $2^{-\Delta \Delta \mathrm{Cq}}$, where $\Delta \mathrm{Cq}=\mathrm{Cq}$ (HOTAIR) - Cq ( $\beta$-actin). The HOTAIR expression levels in tumors were compared to normal tissues. To minimize experimental variability, each sample was analyzed in triplicates and the mean expression levels were calculated.

Plasmids and transfection. Full length human HOTAIR DNA were cloned into a pLVX-CMV-PGK-puro vector (BioWit Technologies Ltd., Shenzhen, China). Recombinant plasmids were used for transfecting cells using Lipofectamine 2000 (Invitrogen Life Technologies, Carlsbad, CA, USA). MDA-MB231 cells were grown in culture media containing 10\% FBS. Lipofectamine 2000, opti-MEM (Gibco Life Technologies, Carlsbad, CA, USA) and plasmid pLVX-CMVHOTAIR-PGK-puro were mixed and incubated for $20 \mathrm{~min}$ to be added to MDA-MB231 cells. On reaching confluency, the MDA-MB231 cells were transfected at $25^{\circ} \mathrm{C}$. After
$6 \mathrm{~h}$ of incubation, the cells were incubated in RPMI-1640 containing $10 \% \mathrm{FBS}$ in $5 \% \mathrm{CO}_{2}$ at $37^{\circ} \mathrm{C}$. After transfection, the cells were subjected to puromycin for selection. After 10 days, the stable HOTAIR transfected MDA-MB231 cell line (HOTAIR-MDA-MB231) was identified. Untransfected cells were used as the blank control, and cells transfected with pLVX-CMV-PGK-puro (empty vector) (vector-MDA-MB231) were used as the negative control.

Clonogenic survival assay. The cells were seeded in 6-well plates for $24 \mathrm{~h}$, subjected to X-rays of 2, 4, 6, and $8 \mathrm{~Gy}$, and then cultured at $37^{\circ} \mathrm{C}$ for 12 days. After incubation, the cells were fixed with methanol and stained with Giemsa. Any colonies containing $\geq 50$ cells were counted under the microscope (BX-51, Olympus, Tokyo, Japan).

Immunohistochemistry. The Ki67 expression level was measured using immunohistochemistry, as previously described (8). Immunohistochemical studies were performed on one representative block, using the avidin-biotin complex horseradish peroxidase (HRP) method. Briefly, the sections were fixed in formalin, embedded in paraffin, and treated with heat-induced epitope retrieval. Ki67 staining was applied by mouse anti-human monoclonal MIB-1 antibody (dilution: 1:100, cat no.: ZM-0165, Beijing Zhongshan Jinqiao Biotechnology Co., Ltd., Beijing, China) according to the manufacturer's guidelines. Blank control (MDA-MB231), negative control (Vector-MDA-MB231) and positive tests (HOTAIR-MDA-MB231) were run simultaneously. Bound secondary antibodies were visualized with diaminobenzidine (Beijing Zhongshan Jinqiao Biotechnology Co., Ltd., Beijing, China) and counterstained with Harris hematoxylin. Samples were examined by two independent pathologists who had no prior knowledge of the clinical data. The expression levels of Ki67 were graded based on the percentage of tumor cells stained by the antibody.

Western blot analysis. Cells were treated with $10 \mu \mathrm{mol} / 1 \mathrm{PI} 3 \mathrm{~K}$ inhibitor (LY294002) for $6 \mathrm{~h}(9,10)$. The cells were lysed in RIPA buffer supplemented with protease and phosphatase inhibitors (Keygen, Nanjing, China). Quantity of protein in the lysates was measured using a BCA kit (Beyotime Institute of Biotechnology, Haimen, China) and equal amounts of proteins were separated by SDS-PAGE and transferred to PVDF membranes (Millipore, Alsace, France). The membranes were blocked with $5 \%$ skim milk, incubated with primary antibodies against HOXD10 (dilution: 1:5,000, cat no. AB76897; Abcam, Cambridge, MA, USA), p-AKT (dilution: 1:300, cat no. KG11054) and p-BAD (dilution: 1:300, cat no. KG11068) (both from Nanjing Keygen) at $4^{\circ} \mathrm{C}$ overnight, and HRP-conjugated secondary antibodies (Bioworld Technology, Inc., St. Louis Park, MN, USA) for $1 \mathrm{~h}$ at $25^{\circ} \mathrm{C}$. Immunoblotted proteins were visualized by ECL reagents and the signals were detected by ChemiDoc ${ }^{\mathrm{TM}}$ XRS imaging system (Quantity One Quantitation software, Bio-Rad Laboratories, Inc., Hercules, CA, USA).

Statistical analysis. Comparisons of continuous data were conducted by the independent test or paired t-test between the groups, whereas categorical data were analyzed by the 


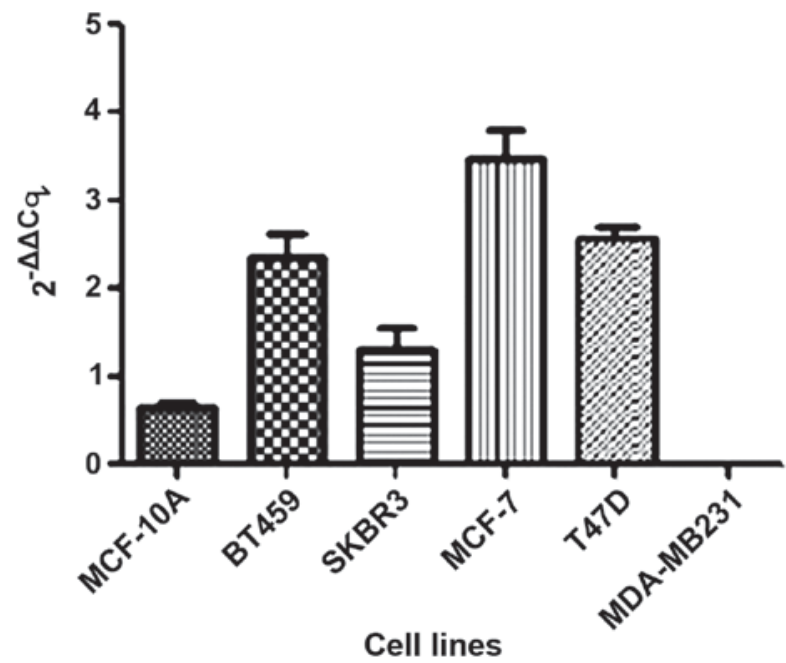

Figure 1. HOTAIR expression in five tumoral (BT549, SKBR3, MCF-7, T47D and MDA-MB231) and one non-tumoral (MCF-10A) breast cell lines detected by reverse transcriptase-quantitative PCR (RT-qPCR). HOTAIR, homeobox transcript antisense RNA.

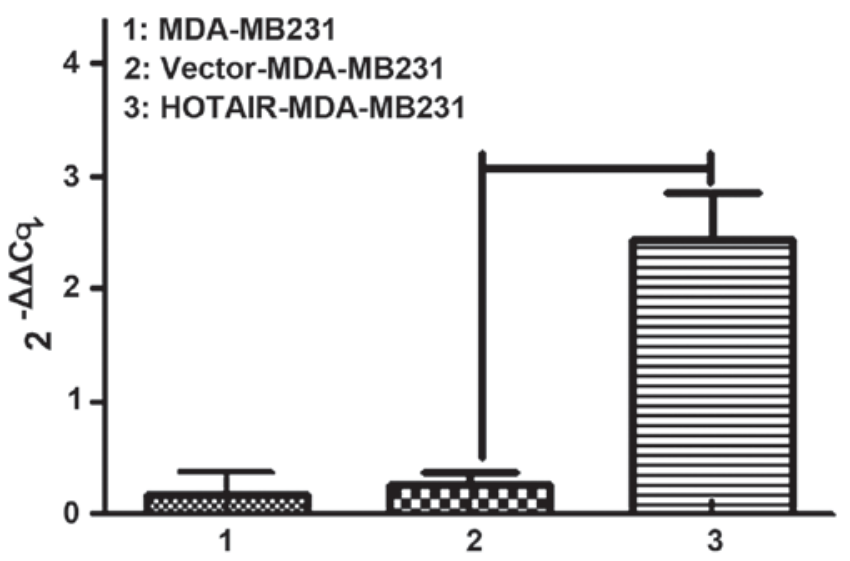

Figure 2. MDA-MB231 cells transfected pLVX-CMV-PGK-puro (HOTAIRMDA-MB231) and pLVX-CMV-HOTAIR-PGK-puro (vector-MDA-MB231). After transfection, three cells demonstrated different levels of HOTAIR. HOTAIR-MDA-MB231 was selected for in vitro experiments. HOTAIR, homeobox transcript antisense RNA.

Chi-square test. Values were shown as the mean $\pm \mathrm{SD}$. Data were analyzed by t-test or one-way ANOVA analysis. The statistical analyses were performed using SPSS for Windows version 16.0 (SPSS, Inc., Chicago, IL, USA) and GraphPad Prism 5 (GraphPad Software Inc., La Jolla, CA, USA). P<0.05 was considered to indicate a statistically significant difference.

\section{Results}

In the present study, we investigated the HOTAIR gene expression in five breast cancer tumor cell lines as well as one non-tumor (MCF-10A) cell line. We observed that HOTAIR expression was lower only in the MDA-MB231 cell line compared to the non-tumoral cell line (Fig. 1).

Thus, MDA-MB231 cells were selected to continue the in vitro experiments. We labeled control and HOTAIR-over-

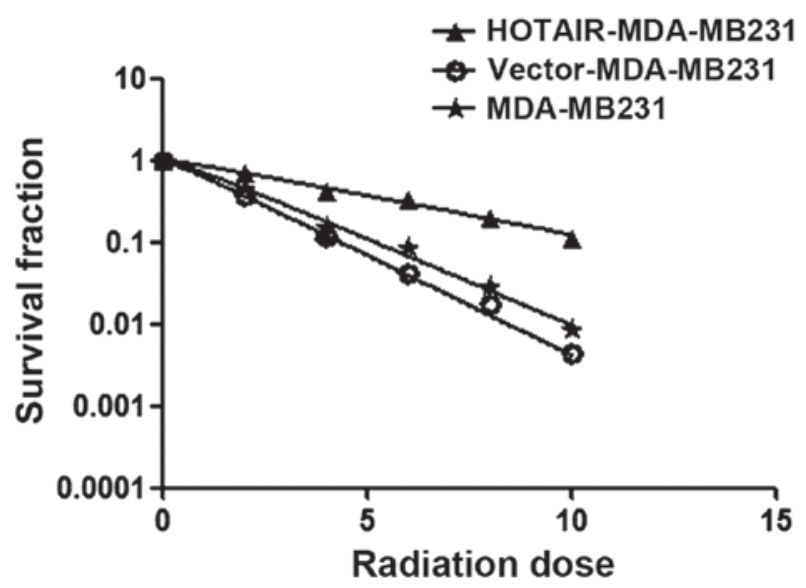

Figure 3. Clonogenic survival assay of MDA-MB231, vector-MDA-MB231 and HOTAIR-MDA-MB231 cells exposed to 0-10 Gy. HOTAIR, homeobox transcript antisense RNA.

Table I. Clonogenic survival assay test, overexpressed HOTAIR accelerated clonogenic survival of MDA-MB231 cells (Ser $=0.58)$.

\begin{tabular}{lcccc}
\hline Cells & D0 & Dq & SF\% & Ser D0 \\
\hline MDA-MB231 & 2.05 & 1.42 & 0.46 & \\
Vector-MDA-MB231 & 1.77 & 1.23 & 0.41 & \\
HOTAIR-MDA-MB231 & 4.46 & 3.09 & 0.71 & 0.58
\end{tabular}

HOTAIR, homeobox transcript antisense RNA.

expressing cells with puro resistance. Lipofectamine 2000 transduction allowed the stable overexpression of HOTAIR to almost 10 -fold over the vector-transduced cells, which were comparable to the levels seen in patients (Fig. 2).

Investigations were performed to verify whether HOTAIR induced radioresistance in MDA-MB231 cells. Using the clonogenic survival assay test, overexpressed HOTAIR accelerated the clonogenic survival of MDA-MB231 cells (Ser=0.58) was examined (Fig. 3) (Table I).

To understand the mechanism controlling HOTAIRinduced radioresistance, the Ki67 expression level was measured. This experiment was performed to determine whether HOTAIR-induced radioresistance was a result of increased apoptosis or proliferation. Null vector transfection (vector-MDA-MB231) served as the control. The results showed that the proportion of apoptotic and proliferative cells was altered $(\mathrm{P}<0.01)$ (Fig. 4).

We also investigated the mechanism underlying the anti-clonogenic effect identified in the overexpressed HOTAIR MDA-MB231 cells. Firstly, we detected HOTAIR expression in postradiotherapeutic breast cancer cells with a minimum level of accumulation at $24 \mathrm{~h}$ (Fig. 5). Thus, the 24-h time point was chosen for subsequent experiments.

In order to investigate whether HOTAIR was involved in HOXD10 expression and the PI3K/AKT-BAD pathway in MDA-MB231 cells, the expression levels of HOXD10, 

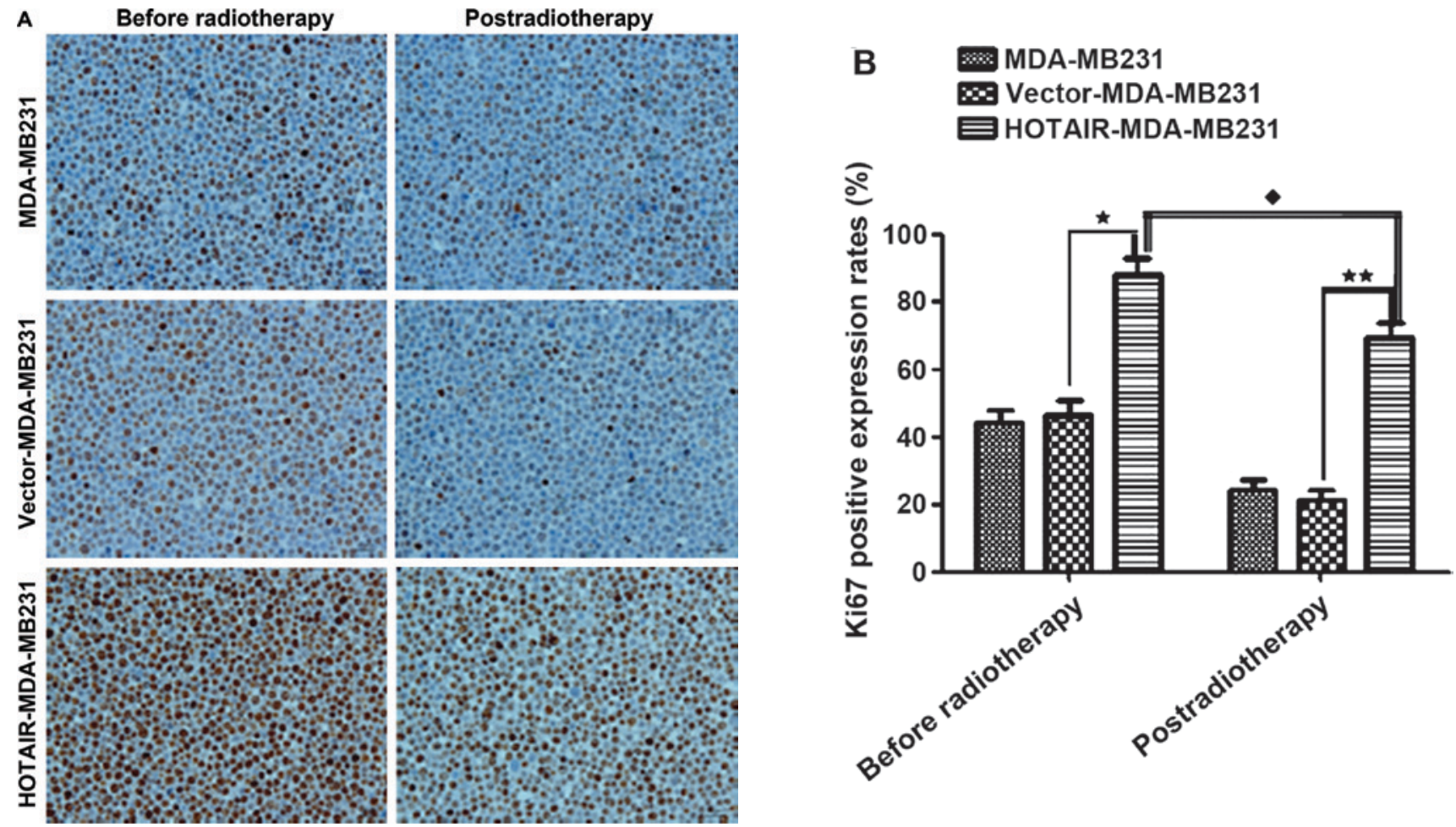

Figure 4. (A) Overexpressed HOTAIR significantly reduced irradiation-induced proliferation in Ki67 using immunohistochemistry. (B) Ki67 of vector-MDA-MB231 had no differences with that of null MDA-MB231 ( $\mathrm{P}>0.05)$. Compared to vector-MDA-MB231 cells, overexpressed HOTAIR cells significantly increased in Ki67 after radiotherapy ("*P<0.05). The Ki67-positive expression rates before and post-radiotherapy were different ("P<0.05). HOTAIR, homeobox transcript antisense RNA.

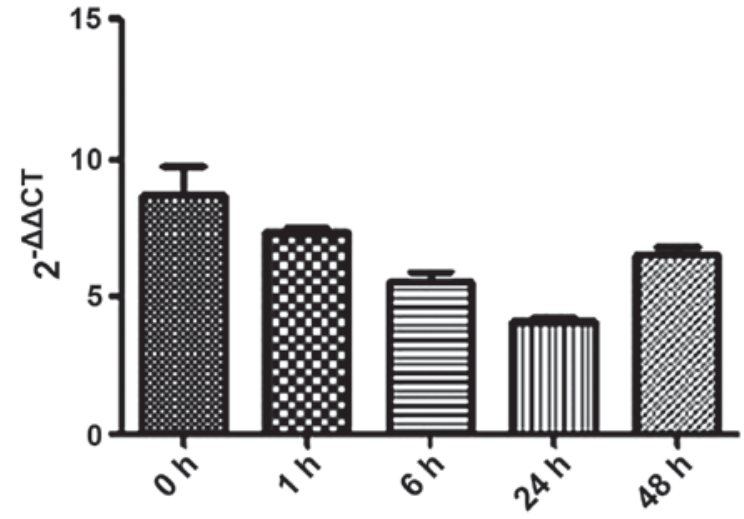

Postradiotherapeutic HOTAIR

Figure 5. Determination of postradiotherapeutic HOTAIR in MDA-MB231 cell foci at 1, 4, 6, 24 and $48 \mathrm{~h}$ after treatment with 8 Gy. HOTAIR, homeobox transcript antisense RNA.
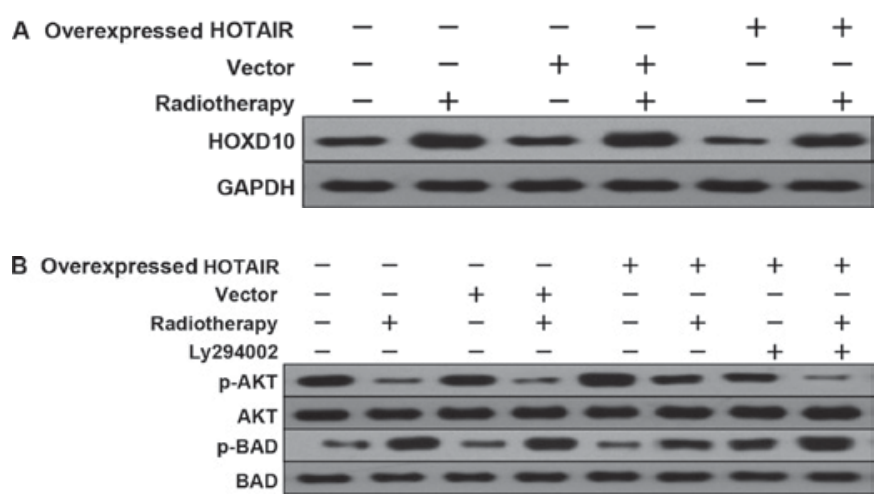

Figure 6. (A) Western blot analysis reveals that HOTAIR led to the suppression of HOXD10 activation, as well as the (B) downregulation of p-AKT and the upregulation of p-BAD expression levels in MDA-MB231 cells. Ly294002 inhibited p-AKT and spontaneously promoted p-BAD. HOTAIR. homeobox transcipt antisense RNA; p-AKT, phosphorylated AKT.

may be a useful strategy for those breast cancers with high expression levels of HOTAIR.

\section{Discussion}

Radiotherapy is the leading method of therapy for inoperable and locally advanced breast cancers. However, breast cancer radioresistance occurs frequently in patients undergoing radiotherapy. LncRNA HOTAIR recently emerged as a cancerogenic promoter in various types of cancer including Therefore, an appropriate dose increase in radiation therapy The results suggested that radiation played an effective
role in the overexpression of HOTAIR breast cancer cells.

phosphorylated AKT (p-AKT) and phosphorylated BAD (p-BAD) were measured. Subsequently, by addin p-AKT and p-BAD proteins was investigated. The results showed that, HOTAIR promoted the proliferation of breast cancer cells via HOXD10 and the PI3K/AKT-BAD pathway (Fig. 6A and B). The results suggested that radiation played an effective
role in the overexpression of HOTAIR breast cancer cells. 
breast cancer (10). Development and metastasis are a common feature shared among some lncRNAs. It has been established that lncRNAs were involved in numerous biological functions including imprinting, epigenetic regulation, apoptosis, and cell cycle (11). It appears that lncRNAs are developing into promising biomarkers and novel strategies against tumor (11). Recent findings on lncRNAs revealed that HOTAIR was expressed from the HOXC locus, whereas HOTAIR transcription was repressed in the more distal HOXD locus (12). The interaction among HOTAIR, PRC2 and the lysine-specific demethylase 1 negatively influenced the expression of certain metastasis-suppressing genes $(3,12)$. Clinically, HOTAIR was significantly overexpressed in a variety of tumors and was shown to induce the proliferation and metastasis thereof (13-18). The overexpression of HOTAIR is a useful predictor of survival and progression in several cancer types, including colon cancer (13), gastrointestinal stromal tumors (14), pancreatic cancer (15), oesophageal cancer (16), hepatocellular carcinoma (17) and nasopharyngeal carcinoma (18). HOTAIR is also involved in the development of primary breast cancers and promotes invasion and metastasis (3).

Our in vitro results demonstrated that the overexpressed HOTAIR in MDA-MB231 cells had a significant effect in cell proliferation. Notably, overexpressed HOTAIR in MDA-MB231 cells maintained higher proliferation rates after receiving irradiation, suggesting that HOTAIR may play a role as a radioresistance factor.

The exact mechanism of HOTAIR-induced radioresistance remains unclear; however, details surrounding the process are emerging gradually. HOTAIR overexpression induced an aggressive phenotype in many cancer types and aberrant expression of homeotic HOX transcription factors, especially HOXD10, which regulated differentiation and tissue homeostasis (19).

Findings suggest that HOXD10 translation can be repressed by two types of ncRNAs: HOTAIR and miR-10b. Liu et al showed that miR-10b promoted cell invasion through the RhoC-AKT signaling pathway by targeting HOXD10 in gastric cancer (20). Previous findings showed that HOXD10 downregulation resulting from miR-10b overexpression may increase prometastatic gene products, such as MMP-14 and RHOC. Additionally, HOXD10 downregulation also contributed to the acquisition of metastatic phenotypes in epithelial ovarian cancer cells (5). In the current study, we investigated the effects of HOTAIR and/or irradiation on increasing HOXD10 and PI3K/AKT-BAD activities. While we observed high levels of HOXD10 expression during radiotherapy, HOXD10 was downregulated in breast cancer cells in which HOTAIR was overexpressed suggesting that, HOTAIR may serve as a limiting factor in HOXD10 expression. Radiotherapy played a central role in the modulation of HOXD10 expression; nevertheless, HOTAIR antagonized and weakened the effects of radiation. Furthermore, HOTAIR negatively affected the BAD protein expression. After radiotherapy, the BAD level increased significantly. BAD expression levels in the overexpressed HOTAIR and radiotherapy group were lower than the null-vector and radiotherapy groups. Our results revealed that after treatment with Ly294002 $(10 \mu \mathrm{mol} / \mathrm{l})$ and radiotherapy, the p-AKT protein levels changed and this change was time-dependent. The results suggest that in breast cancer cells,
HOTAIR had the ability to increase radioresistance by interfering with activation of HOXD10 and the PI3K/AKT-BAD signaling pathway.

We also showed that HOTAIR overexpressions may serve as a predictor to stratify the risk of breast cancer radioresistance. However, which oncogenic signal is responsible for the increase observed in HOTAIR expression in breast tumor cells remains to be determined. Additionally, the role of HOTAIR in promoting proliferation through PI3K/AKT-Bad pathway by targeting HOXD10 should be investigated. In conclusion, our findings reveal that patients with HOTAIR-overexpression breast tumors have a low sensitivity to radiotherapy and adjuvant or more aggressive treatments should be administered to these patients.

\section{References}

1. Ferlay J, Soerjomataram I and Ervik M: GLOBOCAN 2012 v1.0, Cancer Incidence and Mortality Worldwide. IARC CancerBase No. 11 (Internet). International Agency for Research on Cancer, Lyon, France 2013. http://globocan.iarc.fr.

2. Esteller M: Non-coding RNAs in human disease. Nat Rev Genet 12: 861-874, 2011.

3. Gupta RA, Shah N, Wang KC, Kim J, Horlings HM, Wong DJ, Tsai MC, Hung T, Argani P, Rinn JL, et al: Long non-coding RNA HOTAIR reprograms chromatin state to promote cancer metastasis. Nature 464: 1071-1076, 2010.

4. Vardhini NV, Rao PJ, Murthy PB and Sudhakar G: HOXD10 expression in human breast cancer. Tumour Biol 35: 10855-10860, 2014.

5. Nakayama I, Shibazaki M, Yashima-Abo A, Miura F, Sugiyama T, Masuda T and Maesawa C: Loss of HOXD10 expression induced by upregulation of miR-10b accelerates the migration and invasion activities of ovarian cancer cells. Int $\mathbf{J}$ Oncol 43: 63-71, 2013

6. ZLi X, Wu Z, Mei Q, Li X, Guo M, Fu X and Han W: Long non-coding RNA HOTAIR, a driver of malignancy, predicts negative prognosis and exhibits oncogenic activity in oesophageal squamous cell carcinoma. Br J Cancer 109: 2266-2278, 2013.

7. Huuhtanen RL, Blomqvist CP, Wiklund TA, Böhling TO, Virolainen MJ, Tukiainen EJ, Tribukait B and Andersson LC: Comparison of the $\mathrm{Ki}-67$ score and $\mathrm{S}$-phase fraction as prognostic variables in soft-tissue sarcoma. Br J Cancer 79: 945-951, 1999.

8. Hu L, Zaloudek C, Mills GB, Gray J and Jaffe RB: In vivo and in vitro ovarian carcinoma growth inhibition by a phosphatidylinositol 3-kinase inhibitor (LY294002). Clin Cancer Res 6: 880-886, 2000 .

9. Semba S, Itoh N, Ito M, Harada M and Yamakawa M: The in vitro and in vivo effects of 2-(4-morpholinyl)-8-phenyl-chromone (LY294002), a specific inhibitor of phosphatidylinositol 3'-kinase, in human colon cancer cells. Clin Cancer Res 8: 1957-1963, 2002.

10. Zhang J, Zhang P, Wang L, Piao HL and Ma L: Long non-coding RNA HOTAIR in carcinogenesis and metastasis. Acta Biochim Biophys Sin (Shanghai) 46: 1-5, 2014.

11. Tsai MC, Manor O, Wan Y, Mosammaparast N, Wang JK, Lan F, Shi Y, Segal E and Chang HY: Long noncoding RNA as modular scaffold of histone modification complexes. Science 329: 689-693, 2010.

12. Kogo R, Shimamura T, Mimori K, Kawahara K, Imoto S, Sudo T, Tanaka F, Shibata K, Suzuki A, Komune S, et al: Long noncoding RNA HOTAIR regulates polycomb-dependent chromatin modification and is associated with poor prognosis in colorectal cancers. Cancer Res 71: 6320-6326, 2011.

13. Xu ZY, Yu QM, Du YA, Yang LT, Dong RZ, Huang L, Yu PF and Cheng XD: Knockdown of long non-coding RNA HOTAIR suppresses tumor invasion and reverses epithelial-mesenchymal transition in gastric cancer. Int J Biol Sci 9: 587-597, 2013.

14. Kim K, Jutooru I, Chadalapaka G, Johnson G, Frank J, Burghardt R, Kim S and Safe S: HOTAIR is a negative prognostic factor and exhibits pro-oncogenic activity in pancreatic cancer. Oncogene 32: 1616-1625, 2013. 
15. Chen FJ, Sun M, Li SQ, Wu QQ, Ji L, Liu ZL, Zhou GZ, Cao G, Jin L, Xie HW, et al: Upregulation of the long non-coding RNA HOTAIR promotes esophageal squamous cell carcinoma metastasis and poor prognosis. Mol Carcinog 52: 908-915, 2013.

16. Ishibashi M, Kogo R, Shibata K, Sawada G, Takahashi Y, Kurashige J, Akiyoshi S, Sasaki S, Iwaya T, Sudo T, et al: Clinical significance of the expression of long non-coding RNA HOTAIR in primary hepatocellular carcinoma. Oncol Rep 29: 946-950, 2013.

17. Nie Y, Liu X, Qu S, Song E, Zou H and Gong C: Long non-coding RNA HOTAIR is an independent prognostic marker for nasopharyngeal carcinoma progression and survival. Cancer Sci 104: 458-464, 2013

18. Tano $\mathrm{K}$ and Akimitsu N: Long non-coding RNAs in cancer progression. Front Genet 3: 219, 2012.
19. Heubach J, Monsior J, Deenen R, Niegisch G, Szarvas T, Niedworok C, Schulz WA and Hoffmann MJ: The long noncoding RNA HOTAIR has tissue and cell type-dependent effects on HOX gene expression and phenotype of urothelial cancer cells. Mol Cancer 14: 108, 2015.

20. Liu Z, Zhu J, Cao H, Ren H and Fang X: miR-10b promotes cell invasion through RhoC-AKT signaling pathway by targeting HOXD10 in gastric cancer. Int J Oncol 40: 1553-1560, 2012. 